\title{
EXPLORATION OF UNSTEADY AND STEADY CALCULATION METHODS FOR WHEEL SPACE SEALING APPLICATIONS
}

\author{
Alexander Lautenschläger, \\ René Braun, Karsten Kusterer \\ B\&B-AGEMA GmbH \\ lautenschlaeger@bub-agema.de \\ Aachen, Germany
}

\author{
Dieter Bohn \\ RWTH Aachen University \\ dieter.bohn@rwth-aachen.de \\ Aachen, Germany
}

\section{ABSTRACT}

To prevent ingestions of the combustion gas into the cavity between the stationary components and rotating disks, coolant flow from the compressor is used to seal and purge the area against the hot gas. Specific geometries are used to improve the performance of those rim seals in order to save coolant while maintaining a save operation condition for the rotating components. Therefore accurate performance predictions in the early design steps are important to save time and costs. But numerical performance predictions for rim seal applications are still challenging especially for small and mediate enterprises (SME's). Therefore the authors of this paper are looking for methods to reduce time and costs of numerical performance predictions for rim seal geometries, whereas this paper initiates this proposition by investigating the current limits of the available methods for SME's.

This paper compares steady and unsteady RANS (URANS) simulations. The investigations are based on measurement and geometry data taken from a test rig design of a single stage test turbine at RWTH Aachen University. The RANS simulations did not show a significant ingestion of hot gas whereas the URANS approach has shown increased hot air ingestion. In order to achieve a converged flow field in the URANS simulations, a large number of full rotation cycles have to be calculated. The results show, that pressure oscillations driven by the blade pressure field, as well as acoustics frequencies have been captured by the URANS approach.

Nevertheless the investigated URANS simulation approach still shows significant deviation in the prediction of the hot gas ingestion compared to the experimental test data. This is due to the fact that the fundamental equations and the applied closure by isentropic turbulence models are not capable of resolving sufficiently characteristic flow phenomena inside the wheels space and the sealing gap.

Another limitation is by the chosen sector model with regard to flow phenomena in circumferential direction.

\section{NOMENCLATURE}

$a_{m}$

$C_{W}$

$c_{1}$

$d$

$f$

$f_{B}$

$f_{V}$

$J_{n}$

$M a_{1}$

$\dot{m}_{S A S}$

$n$

$\omega$

$p$

$p_{0}$

$p_{1}$

$p_{t}$

$R$

$R e_{c 1}$

$R_{\text {Lip }}$

$R_{S}$

$R e_{U}$

$R_{V}$

$r$ $\frac{m}{s} \quad$ Speed of Sound

- Non-dimensional mass flow rate

$\frac{m}{s} \quad$ Velocity magnitude at surface 1

$\mathrm{mm}$ Distance NGV trailing edge to seal gap

$\mathrm{Hz}$ Frequency

$\mathrm{Hz} \quad$ Blade passing frequency

$\mathrm{Hz}$ Resonance frequency of a volume

- Bessel function of order $n$

_ Mass flow averaged Mach number a surface " 1 "

$\frac{\mathrm{kg}}{\mathrm{s}} \quad$ Sealing mass flow rate

Rotational frequency of the rotor

Rotational rate of the rotor

bar Static pressure

bar Inlet static pressure

bar Static hub pressure at surface 1

bar Total pressure

$\mathrm{mm}$ Hub radius

Mass flow averaged Reynolds number at surface " 1 "

$\mathrm{mm}$ Lip radius

$\frac{J}{\operatorname{kgK}}$

Specific gas constant

Mass flow averaged circumferential Reynolds number

$\mathrm{mm}$ Volume radius

$\mathrm{mm}$ Radial machine coordinate 


\begin{tabular}{|c|c|c|}
\hline$s$ & $\mathrm{~mm}$ & Wheel space clearance \\
\hline$s_{c}$ & $\mathrm{~mm}$ & Seal gap clearance \\
\hline$T_{1}$ & $K$ & Static Temperature at surface 1 \\
\hline$T_{t}$ & $K$ & Total Temperature \\
\hline$t$ & $\circ$ & $\begin{array}{l}\text { Circumferential length of the sector model } \\
\text { at } \mathrm{R}\end{array}$ \\
\hline$z$ & $\mathrm{~mm}$ & Axial machine coordinate \\
\hline$\alpha_{n m}$ & - & Root of the Bessel function $J_{n}$ \\
\hline$\xi_{C A}$ & - & Mass fraction of cavity air (CA) \\
\hline$\kappa$ & - & Specific heat ratio \\
\hline$\mu_{1}$ & $\operatorname{Pas}$ & Dynamic viscosity at surface 1 \\
\hline$\mu_{S A S}$ & $\operatorname{Pas}$ & Dynamic viscosity at SAS inlet \\
\hline$\rho_{1}$ & $\frac{\mathrm{kg}}{\mathrm{m}^{3}}$ & Density at surface 1 \\
\hline$\rho_{S A S}$ & $\frac{\mathrm{kg}}{\mathrm{m}^{3}}$ & Density at SAS inlet \\
\hline$\theta$ & $\circ$ & Tangential machine coordinate \\
\hline FVV & & $\begin{array}{l}\text { German Research Assoziation } \\
\text { „Forschungsvereinigung Verbrennungs- } \\
\text { kraftmaschinen e.V.“ }\end{array}$ \\
\hline NGV & & Nozzle Guide Vane \\
\hline LES & & Large Eddy Simulation \\
\hline RANS & & Reynolds-average Navier-Stokes \\
\hline SAS & & Secondary Air System \\
\hline URANS & & $\begin{array}{l}\text { Unsteady Reynolds-average Navier- } \\
\text { Stokes }\end{array}$ \\
\hline NGV-TE & & Nozzle Guide Vane Trailing Edge \\
\hline
\end{tabular}

\section{INTRODUCTION}

Stationary and aero engine gas turbines are in constant need of improving the efficiency in order to provide an increased operation value and a decreased environmental cost. An increased focus is given on the secondary air system, which provides critical cooling and sealing capabilities to the machine, but also debits the efficiency.

One of these cooling applications is the sealing flow between the stationary components of the guide vanes and the fast spinning rotor. In order to seal the cavity between the rotating disk and the stationary components from the hot main flow, a certain amount of purge flow is extracted from the compressor and guided through the cavity into the main flow.

In order to provide a sufficient cooling and sealing performance and keep the purge flow rate as minimum as possible, one must know the driving flow mechanism, which lead to the ingress of hot gas from the main flow into the wheel space sealing.

Investigations on sealing characteristics between a rotating and stationary disk reach back to 1970, when Bayley and Owen (Bayley, et al., 1970) published their work of a rotating disk with a simple axial seal gap between rotor and stator. The air was discharged into the environment without any external geometry or main flow. Though no external influence was present, the observations captured the ingestion of atmospheric matter into the wheel space. The ingestions caused by rotational effects are labeled as Rotationally Induced Ingress (RI). Bayley and Owen developed a linear correlation for the determination of the minimum no dimensional flow rate $\mathrm{C}_{\mathrm{w}, \min }$.

Phadke and Owen (Phadke, et al., 1988a), (Phadke, et al., 1988b), (Phadke, et al., 1988c) continued investigations in various seal geometries with and without the presents of the main flow and captured external induced ingress (EI) as well as RI. Additionally an improved linear correlation was developed to determine the minimum non-dimensional mass flow rate. Hamabe and Ishida (Hamabe, et al., 1992) defined correlations for EI ingress as well, with their measurements of a turbine stage with NGV's and no rotor airfoils. Bohn and Wolff (Bohn, et al., 2003) developed an improved correlation for the sealing effectiveness and correlated a linear behavior for 4 basic sealing configurations.

To improve correlations and predictions of the sealing performance in general, it is important to capture the highly unsteady behavior of the seal gap as well as wheel space area. Interaction of the NGV's wake with the pressure field of the rotor airfoils enhances complex flow pattern. Jakoby et al (Jakoby, et al., 2004) did valuable numerical investigations with support of experimental data of the ICAS-GT2 test rig, located at the RWTH Aachen University. The calculations were able to capture large scale unsteady phenomena inside the wheel space volume. The measurements indicated pressure fluctuations depending on the non-dimensional mass flow rate $\mathrm{C}_{\mathrm{w}}$ and below the frequency of the blade passing.

Further numerical studies on observing those pressure fluctuations are present in Boudet et al (Boudet, et al., 2006) publication as well as Rabs et al (Rabs, et al., 2012), who performed a parameter study to investigate the so called Kelvin-Helmoltz instabilities. LES and unsteady RANS studies in challenge with measurements done by Gentilhomme et al (Gentilhomme, et al., 2003) were executed by O'Mahoney et al (O'Mahoney, et al., 2010) with the conclusion that the fidelity of the turbulence model is of greater importance. The LES model performed better compared to the unsteady RANS simulation, both of which only considered a sector of the machines annulus. An additional $360^{\circ}$ model was run with the unsteady RANS turbulence model, with no observed effect on the ingestion behavior. Though O'Mahoney points out, that the simulation time may have not been sufficient enough to conclude the importance of a full-scale geometry.

Recent LES investigations of a $360^{\circ}$ single stage turbine, performed by Pogorelov et al (Pogorelov, et al., 2018), showed outstanding agreements with measurements of Bohn et al (Bohn, et al., 2000) in the prediction of hot gas concentrations inside the wheel space area. Highly unsteady flow phenomena and large scale pressure modes have been captured.

Since these high fidelity calculations are too expensive for computation resources of small and medium enterprises (SME's), it is important to investigate the limits of feasible approaches and to consider them in the design approach. This paper covers steady and unsteady RANS calculations of the measurements of Bohn et al (Bohn, et al., 2000). The target 
is to create a foundation for further investigations to exploit the limits of current SME simulation approaches and develop more accurate methods.

\section{TEST RIG}

The test rig used for the simulations was located at the RWTH Aachen University and run by the Institute for Gas and Steam Turbines. The rig was build and developed in national research projects initiated by the German research association „Forschungsvereinigung Verbrennungskraftmaschinen e.V. (FVV). In those projects the machine was additionally equipped with a helicopter rotor stage to investigate the sealing behavior under more realistic conditions. Followed by a third research project, further sealing configurations were investigated, upon which the current calculations in this paper are based. Bohn et al (Bohn, et al., 2000) published measurements of the cooling air concentrations inside the wheel space for two different seal configurations parallel with an unsteady RANS calculation. Based on the national research projects, Bohn and Wolff (Bohn, et al., 2003) published an improved formulation to predict the minimum sealing flow $\mathrm{C}_{\mathrm{w}, \min }$ with a minimum of three sampling points.

To get a better understanding of the test section, a cross sectional view of the single stage test rig is displayed in Figure 1. The red area indicates the main flow moving from left to right. Passing the $30 \mathrm{NGV}$ airfoils, the main flow meets with the purge flow, which is entering upstream the rotor shaft and exits through the 62 rotor airfoils. The purge air flow, blue area and containing 5\% CO2, enters turbine stage as highlighted by the enlarged detail of the single axial lip rim seal configuration

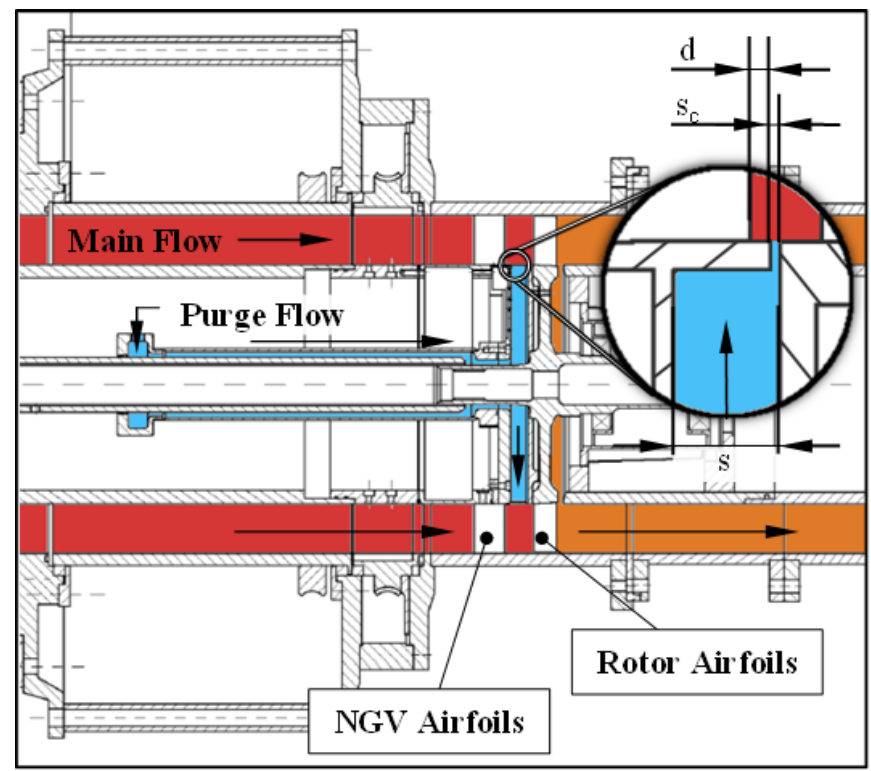

Figure 1: Wheel-Space Sealing Test Rig at the RWTH Aachen (Bohn, et al., 2003)

The measurements used as benchmark for the simulations are concentration measurements of $\mathrm{CO} 2$ at extraction holes on the stator surface. A reduction in $\mathrm{CO} 2$ concentration from the 5\% purge flow supply level is indicative of hot gas ingestions.

\section{SIMULATION DOMAIN}

The simulation domain, pictured in Figure 2, contains one vane section of the flow path in order to reduce the numerical effort. This approach is commonly used in industrial projects, since the computation effort for full scale models are too expensive. The rotating domain of the unsteady RANS model contains two rotor airfoils, which allow a smooth transition from the vane to the blade domain due to an equal sector angle. The interface between the stationary and rotating mesh domains are realized via a repeating interface. The steady RANS approach uses one rotor airfoil, with an implemented mixing plane between the stationary and rotating reference frame domain.

The inlet surface is labeled as " 0 " where total pressure and total temperature is prescribed. In order to differentiate the main flow from the secondary air flow, the inlet flow at surface 0 is marked with a Tracer as "Hot Gas". Together with the pressure outlet surface " 2 ", the conditions are adjusted to meet the Reynolds number and Mach number at the surface plane " 1 ". The purge flow is setup as a mass flow inlet and labeled as SAS. The flow composition consists of $5 \% \mathrm{CO} 2$ and $95 \%$ of Air. The matter in total is marked as "Cavity Air" by the Tracer. The boundary conditions at the stagnation inlet and mass flow inlet are adjusted to match the reported operation conditions listed in Table 1. The main flow is constrained by the mass flow averaged Reynolds and Mach number at Position 1. The purge flow is defined by the non-dimensional mass flow $\mathrm{C}_{\mathrm{w} \text {,min }}$ and the rotational Reynolds number $\operatorname{Re}_{\mathrm{U}}$.

$$
\begin{array}{ll}
R e_{c 1}=\frac{c_{1} \rho_{1} R}{\mu_{1}} & =8 \cdot 10^{5} \\
M a_{1}=\frac{c_{1}}{\sqrt{\kappa R_{S} T_{1}}} & =0.37 \\
R e_{U}=\frac{\rho_{S A S} \omega R^{2}}{\mu_{S A S}} & =8 \cdot 10^{5} \\
C_{W}=\frac{\dot{m}_{S A S}}{\mu_{S A S} R} & =2000
\end{array}
$$

Table 1: Operation Condition

Two different time models are investigated; the steady RANS and the unsteady RANS approach. The turbulence models for the steady and unsteady RANS approach was chosen to be the $\mathrm{k}-\omega$-SST. The time resolution of the unsteady RANS approach equals 0.5 degrees of the rotor progression and the temporal discretization is of 2nd-order. For initialization of the unsteady simulation, the steady RANS calculation was used. The simulations used an all y+ Wall treatment. The $y+$ range is for the airfoil wall, stator and rotor disk wall and the stator and rotor hub surface near the sealing lip below 1. Surfaces which are not close to the sealing area are not covered with prism layer and therefore $y+$ values exceed to values of 100 . 


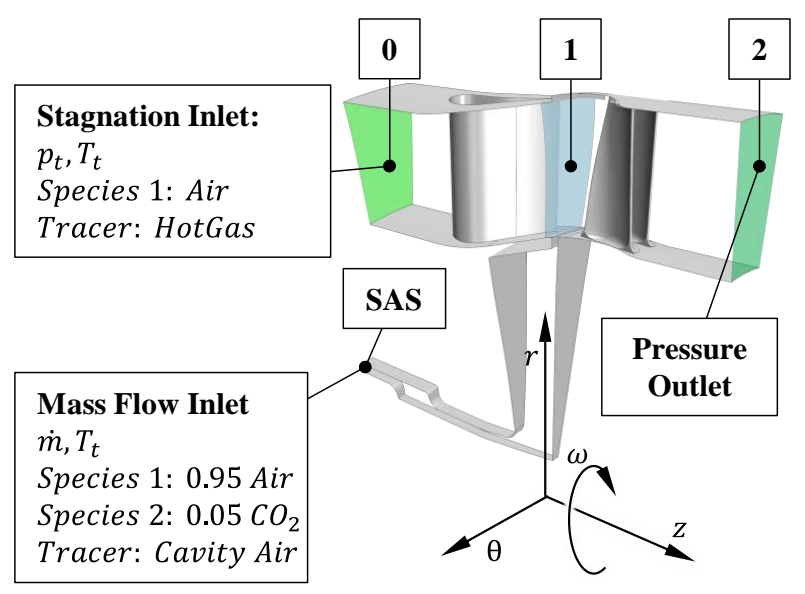

Figure 2: Simulation Domain with Boundary
Conditions

\section{MESH}

The polyhedral mesh, implemented in STAR-CCM+, is used for all calculations and provides meshing for complex geometries. The Mesh is displayed in Figure 3 on the surfaces of the fluid geometry and in the volume extraction through the main flow path and the sealing area.

The mesh approach in these calculations was focused on refinements in areas which are important to determine the sealing performance. Considering the importance of the pressure distribution, formed by the trailing edge and profile losses, the Airfoil of the NGV was equipped with prism layer and an increased resolution compared to the base size of the main flow path. Since the interaction of the rotor airfoil pressure field in front of its leading edge with the wake of the NGV airfoil is highly important for the pressure distribution at the sealing lip, the gird resolution in between this area is increased as well.

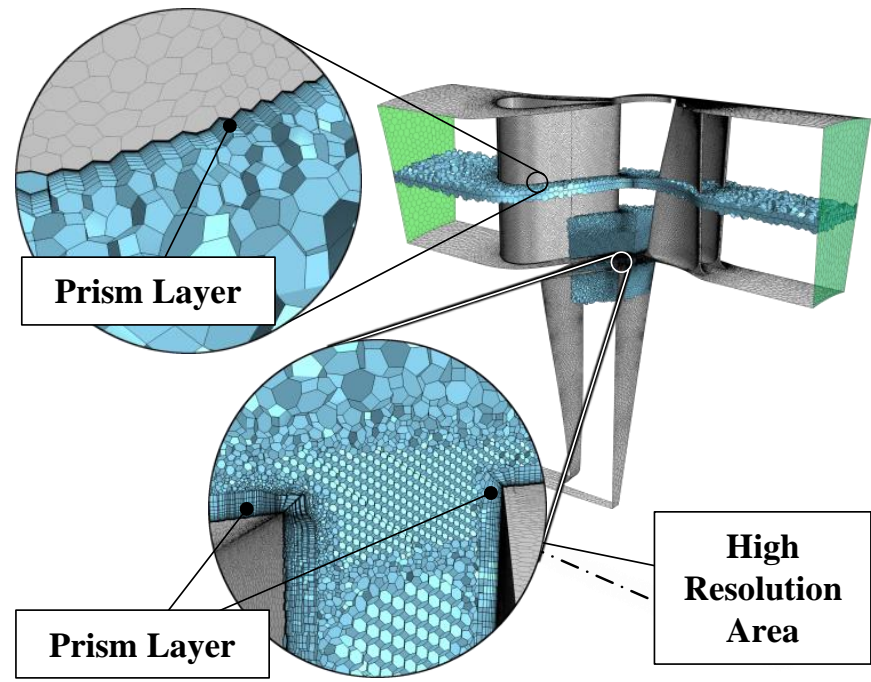

Figure 3: Mesh with Coarse Resolution Settings

In order to capture the complex cross flow right at the sealing lip, a high resolution area was implemented and detailed in Figure 3. The hub surface and of the NGV and the stationary wheel space wall was treated with prism layers to capture the boundary layer flow towards the sealing clearance and to provide a sufficient amount of cells to capture a possible ingress along the NGV's wheel space wall. The rotating wheel space surface was equipped with prism layer as well, since the development of a sufficient boundary layer is important for the outflowing cavity along the rotor surface. The shear layer between the crossing flows and the 3 dimensional vortex structures at the sealing clearance are ascertained with small polyhedral cells in this area. The resolution of the mesh decreases radially inwards, but is still noticeable higher than the main flow.

The total cell count for the unsteady RANS calculation is 2.5 million cells. For the RANS calculation, three meshes have been investigated, where the coarse mesh mirrors the resolution settings of the unsteady RANS calculation. The medium and fine cell counts are 6.2 and 15.1 million and are scaled via the base size, since all custom surface controls are defined relative to its base size. The mesh composition for the performed calculations is summarized in Table 2.

\begin{tabular}{lccc}
\hline Mesh & Coarse & Medium & Fine \\
\hline Million Cells & 2.5 & 6.2 & 15.1 \\
RANS & $\bullet$ & $\bullet$ & $\bullet$ \\
URANS & $\bullet$ & - & - \\
\hline
\end{tabular}

Table 2: Mesh Composition

\section{MESH INFLUENCE}

One of the results extracted in this paper are circumferentially averaged mass fraction concentrations at specific radial locations on the stator surface disk. These locations are displayed in Figure 4 and match the radial locations of the measurement positions of the test rig. The measurements of Bohn et al (Bohn, et al., 2000) represent concentration measurements via extraction holes, which are circumferentially distributed on these locations.

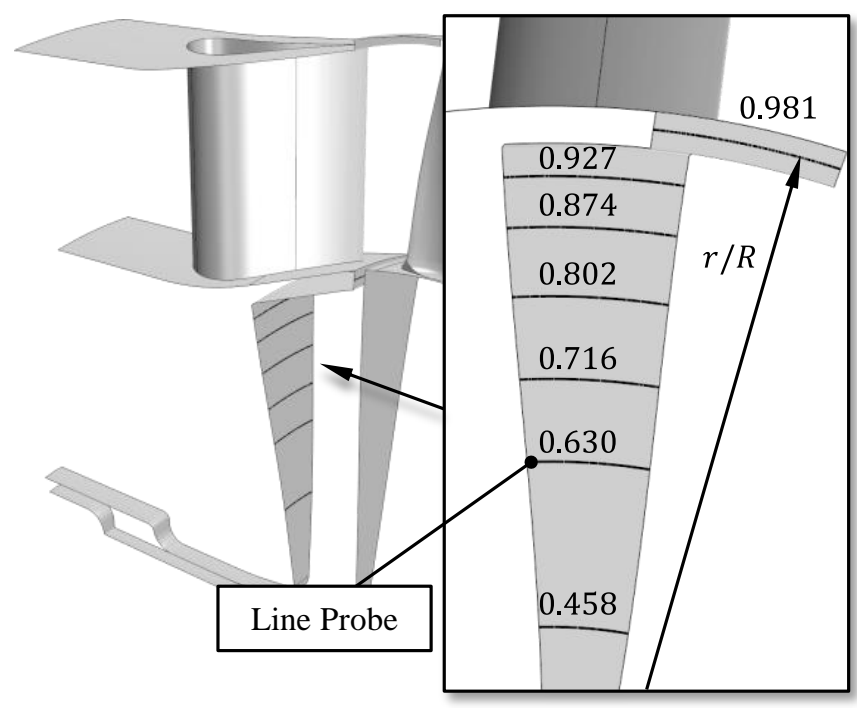

Figure 4: Evaluation positions for the mass fraction distribution $\xi_{\mathrm{CA}}$ and transient pressure development 
The experimental and numerical distributions of the mass fraction of cavity air $\left(\xi_{\mathrm{CA}}\right)$ are displayed in Figure 5 and Figure 6 . The black dots represent the measured values of the experiment whereas the green dots are the results of the unsteady RANS simulation. The connection lines are included for visibility reasons. The uncertainties of the $\mathrm{CO} 2$ measurement were addressed by Bohn at al. (Bohn, et al., 2000), (Bohn, et al., 2003) and have been estimated to be $\pm 2 \%$. The uncertainty is displayed in Figure 5 with red limiter. The extracted graphs of the URANS calculation each represent a different progression in time. The first extraction was done after 1.2 rotations of the rotor domain and indicates a certain amount of hot gas ingress into the seal lip area at $\mathrm{r} / \mathrm{R}=0.981$. Compared to the experimental results the URANS calculation deviates in the prediction of the mass fraction by 41 percentage points at $\mathrm{r} / \mathrm{R}=0.981$.

The positions inside the wheel space area $(0.927-$ 0.458 ) do not show any ingestion signs after 1.2 rotations. With further progression in time, the mass fraction of cavity air $\left(\xi_{\mathrm{CA}}\right)$ decreases noticeably up to the rotation count of 24.7 rotor rotations inside the wheel space area, whereas the seal lip area converged at earlier time periods. After 24.7 rotations one can still see a noticeable deviation to the experimental results. By comparing the gradients of the results, the URANS does show a quite good match in the radial range of $r / R \in$ [0.981:0.716]. In lower radial regions the gradient of the measured results indicates an increase growth compared to the URANS results.

To conclude a general statement about the capability of the URANS calculation, it is important to identify the reasoning behind the deviations from the measurements.
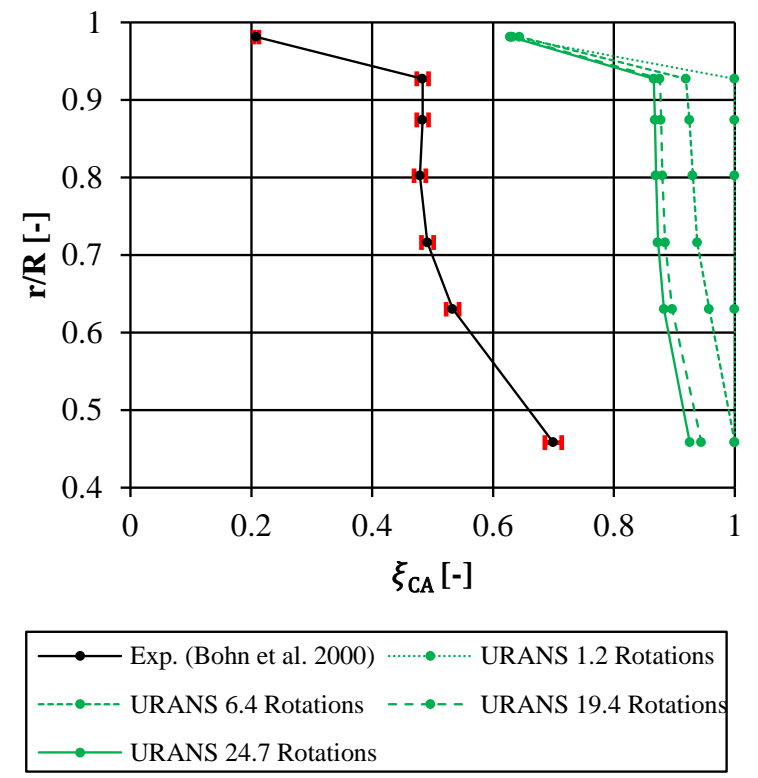

Figure 5: Development of the Mass Fraction of Cavity Air Distribution inside the Wheel Space area for the unsteady RANS approach

One of the first uncertainties, which will always be questioned, is the sufficient resolution of the calculation grid. Since a 2.5 million grid count is a coarse resolution of the fluid domain, considering the current research standards, a mesh study is needed. In order to investigate a first mesh sensitivity, the steady RANS approach was used to perform calculations with the meshes "Medium" and "Fine". The sensitivity approach with the steady RANS model was chosen due to the lower computational effort and to receive a first insight into the difference between the steady and unsteady phenomena.

The mass fraction distribution of the steady RANS calculations in comparison with the URANS calculation is displayed in Figure 6. As already familiar the URANS results are the green dots. The blue, red and violet dots are the results for the coarse, medium and fine RANS calculations.

By comparing the mass fractions at $r / R=0.927$, one can see that all steady RANS calculations show less hot gas ingress, which is expected, since the vane-blade pressure field interaction is not covered in steady RANS calculations, due to the implementation of the mixing plane. But the important behavior, which is observable in that figure, is the decrease in hot gas concentration with the increase in mesh size.

The decrease in mass fraction of cavity air, from the coarse RANS calculation to the fine RANS calculation is about 0.12 at $\mathrm{r} / \mathrm{R}=0.981$. If one can assume, that the mesh sensitivity of the steady RANS calculation can be transferred to the URANS approach, the deviation of the URANS calculation to the measurements would be reduced to 0.29 at that location. The next uncertainty would be the amount of decrease inside the wheel space $r / R \in$ [0.927:0.458]. It is likely that the wheel space concentration changes to an equivalent amount and not only by 0.012 , like the steady calculations show. Since the steady approach does not cover the time depending phenomena happening in the seal gap. Due these observations, a mesh study with the URANS method needs to be performed.

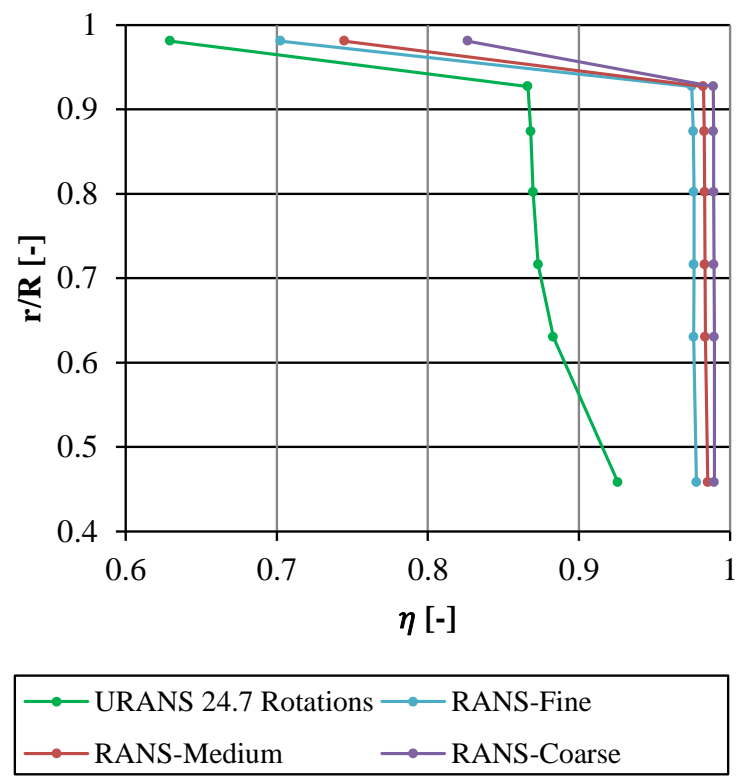

Figure 6: Mass fraction of cavity air distribution inside the wheel space area for the unsteady and steady RANS approach 
The distribution in the wheel space region does show very little ingress for all RANS meshes. Additionally a gradient in the space of $r / R \in$ [0.458:0.716] is not observable. Since the amount of hot gas transferred into the wheel space, is dependent on the concentration of the seal lip area, it would be reasonable to assume the wheel space area would increase in equivalent way. But the difference in concentration between the meshes is relatively small. The ingestion behavior is heavily dependent on the pressure distribution behind the vane and the flow field characteristic in the sealing and wheel space area. Therefore a comparison of these conditions will be covered next.

The circumferential normalized pressure distribution in Figure 7 is extracted at the vane hub surface $1.5 \mathrm{~mm}$ downstream of the vane's trailing edge. The tangential position is further normalized with the radian measure of the hub surface length in circumferential direction. The position $\theta / t=0.0$ was then shifted to match the tangential position of the vane trailing edge. The distribution shows the characteristic maximum and minimum peak of a NGV stage. The maximum peak indicates the wake of the vane trailing edge, whereas the minimum peak shows the location of the flow path main stream. This pressure difference is one of the causes for an inhomogeneous out flow characteristic of the purge flow, into the main flow.

The comparison between the three different RANS calculations does visualize a decrease of the pressure level with the increase in mesh size. The peak to peak pressure difference at the same time stays nearly equal. The distribution of the URANS calculation matches the peak pressure of the coarse RANS calculation quite well, while deviating slightly around those peaks.

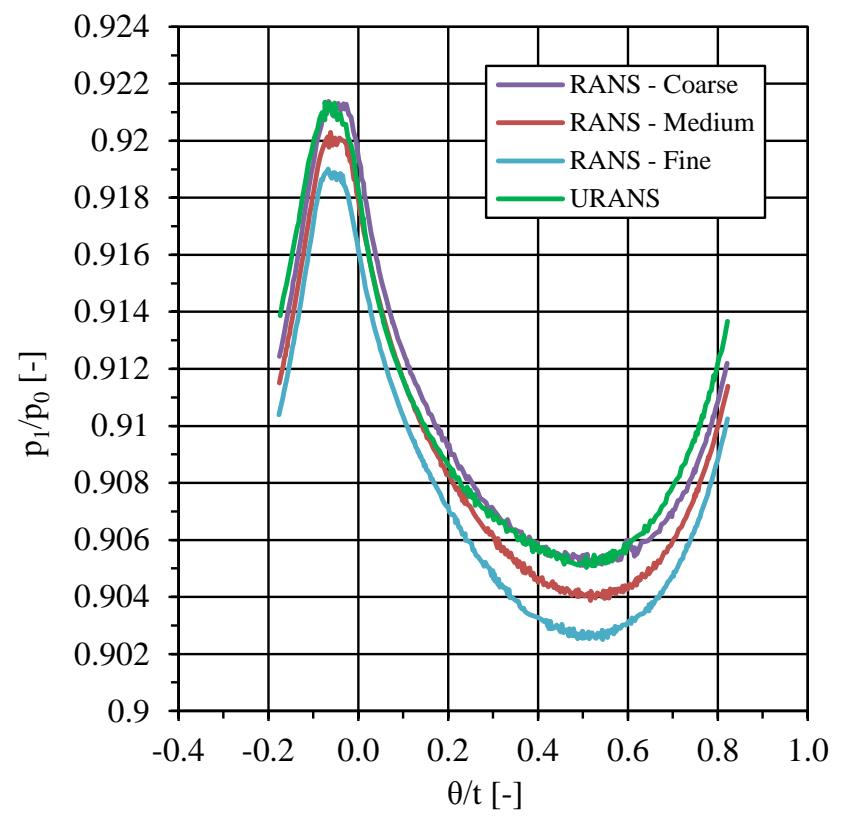

\section{Figure 7: Normalized pressure distribution 1.5mm downstream of the vane's trailing edge}

Moving on with a closer look into the hot gas convection through the stator boundary layer, one can focus the attention to Figure 8. This graphic visualizes the contour plot of $\xi_{\mathrm{CA}}$ on the stator disk surface. The view point is in upstream direction with a small tilt around the tangential axis, in order to observe the inner radius of the seal lip. The NGV trailing edge is visible and pointed out.
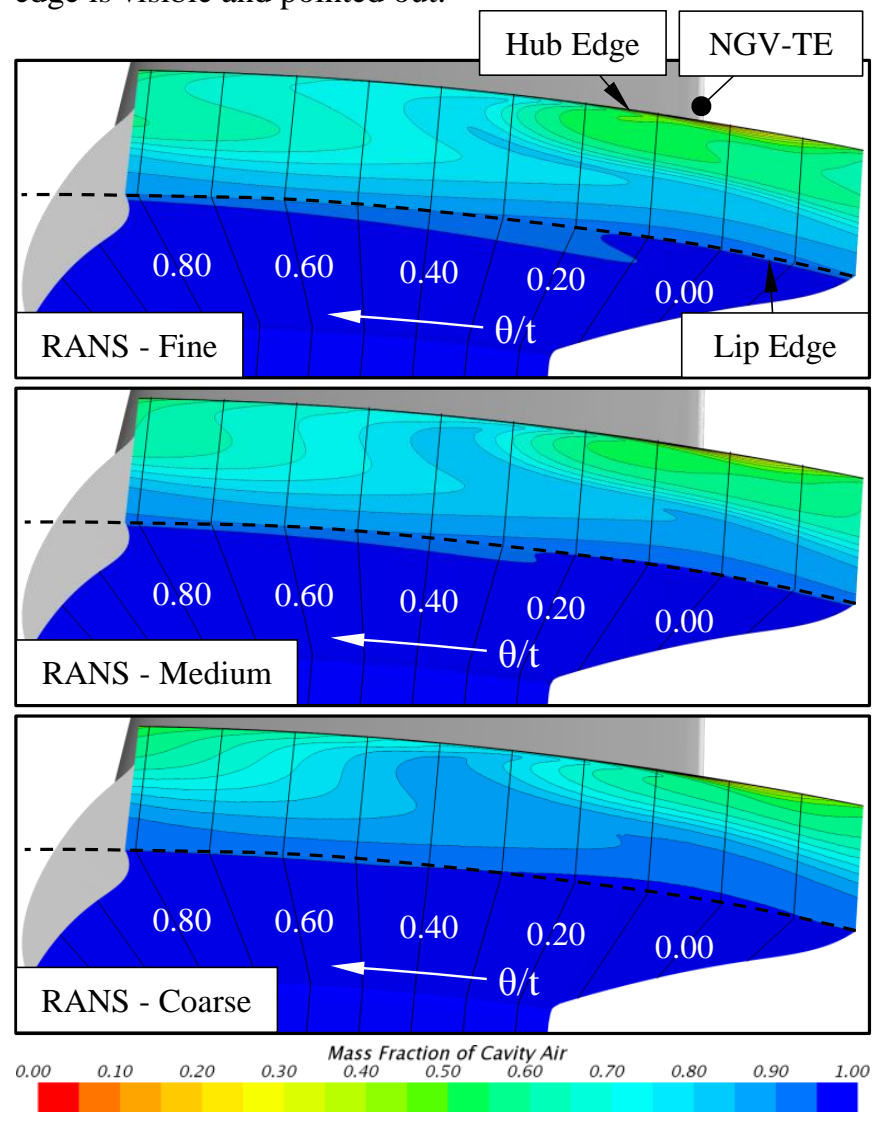

Figure 8: $\xi_{\mathrm{CA}}$ distribution on the stator disk surface

Below the "Hub Edge" one can notice that the hot gas concentration for $\theta / t \notin[0.30: 0.70]$ is significantly increased. These areas show mesh sensitivity, where the fine mesh indicates the highest concentrations of hot gas. Additionally an extension of ingestion regions into the lower radial region is observable as well as ingestion under the lip edge. Even though the ingestion is quite small, it can explain the small increase in hot gas in the wheel space compared to the coarse mesh. At $\theta / t \in$ [0.30:0.70], one can observe an increase in cooling air concentration, which is diminishing with higher mesh resolution.

The cause of the local increase in $\xi_{\mathrm{CA}}$ will be explained with the following Figure 9. The figure displays the flow pattern inside the seal clearance of the coarse RANS calculation. Visualized are the projected streamlines onto surface planes pitched by $\theta / t$ and colored in the passive scalar distribution. The figure shows a recirculation zone, which is occupying a big portion of the circumferential space. The recirculation zone is transferring hot gas from the main flow into the seal clearance and transports the hot matter in negative $\theta /$ t. Due to its positive rotation (Detail B), the hot gas is ingesting near the rotating rotor surface and mixes with the cavity air coming out of the wheel space volume along the stator surface. Moving into the negative tangential direction, the recirculation vanishes and the mixed air is pushed out of the sealing clearance. In the lower half region, 
one can see at the positions $\theta / t \in[0.50: 0.80]$ another recirculation zone, where cavity air is flowing from the wheel space area into the sealing clearance, and turning back again. This recirculation is responsible for the transport of hot gas into the wheel space volume, detailed in A.

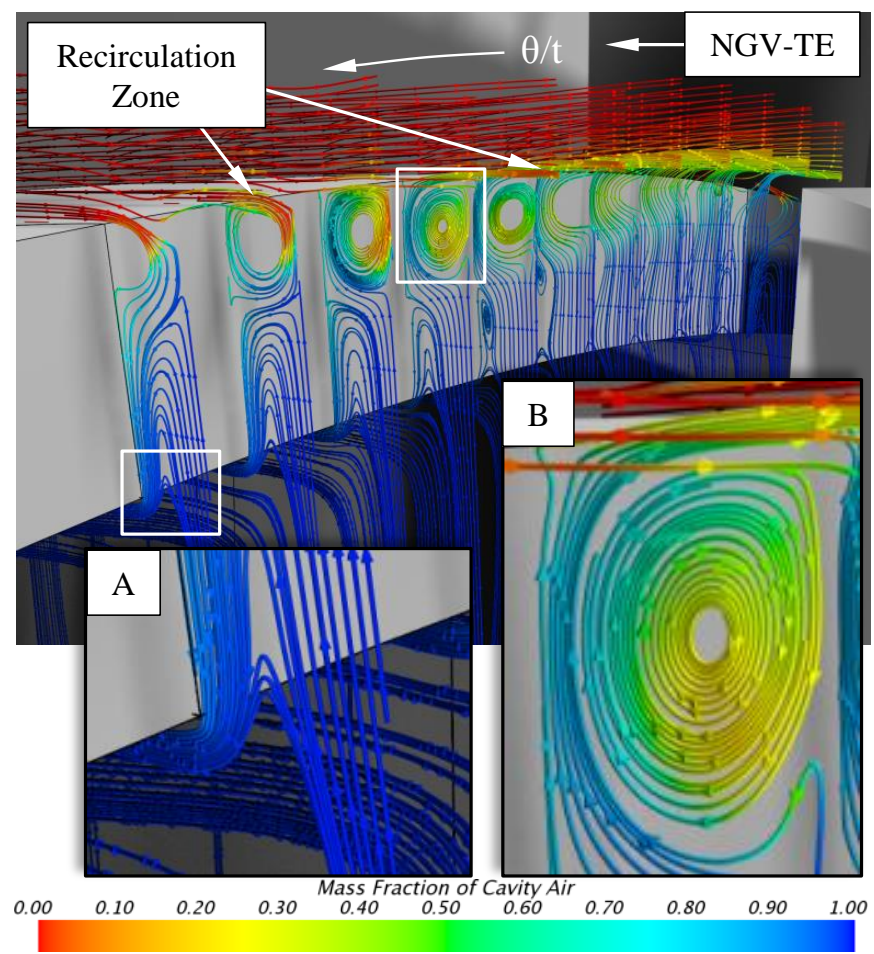

Figure 9: Seal flow field structure of the RANS calculation

The back flow at the stator wall shows a direct flow path into the wheel space volume along the seal lip surface. The back flow is directly connected to the ingestion at the hub edge and thus is getting fed with hot gas. This flow pattern is equivalent for all three meshes. The difference is the already discovered increase concentration of hot gas in the stator boundary layer resulting in a slightly increased ingestion along the lip surface into the wheel space. Since the difference to the experimental results is significant, this flow pattern does miss important features and therefore it does not represent the actual physics of the experiment.

\section{UNSTEADY PHENOMENA}

To evaluate the influence of an unsteady calculation we continue to investigate the flow field at the sealing clearance. Figure 10 visualizes the flow field at the already familiar position at a specific point in time. It is well captured that the flow field is a lot different compared to the steady approach, but certain characteristics remained. For example the recirculation zone, that pulls the hot gas into the sealing lip area, is located at a different position (detail B). The recirculation is moving into the negative circumferential direction as well. This recirculation, reflected at the periodic boundary, transfers the ingested hot gas directly into the lower region of the sealing at around $\theta / t \in$ [0.50:0.80] and further into the wheel space volume as detailed in A. It is well captured that the hot gas transfer into the wheel space is distributed across the axial span of the seal clearance, unlike in the steady RANS calculations. In general the clearance volume experiences an increased hot gas concentration. The flow pattern which leads to the ingestion in detail A does change periodically in concentration of $\xi_{\mathrm{CA}}$ and circumferential location while the progression of physical time. Thus it has to be related to an unsteady phenomenon.

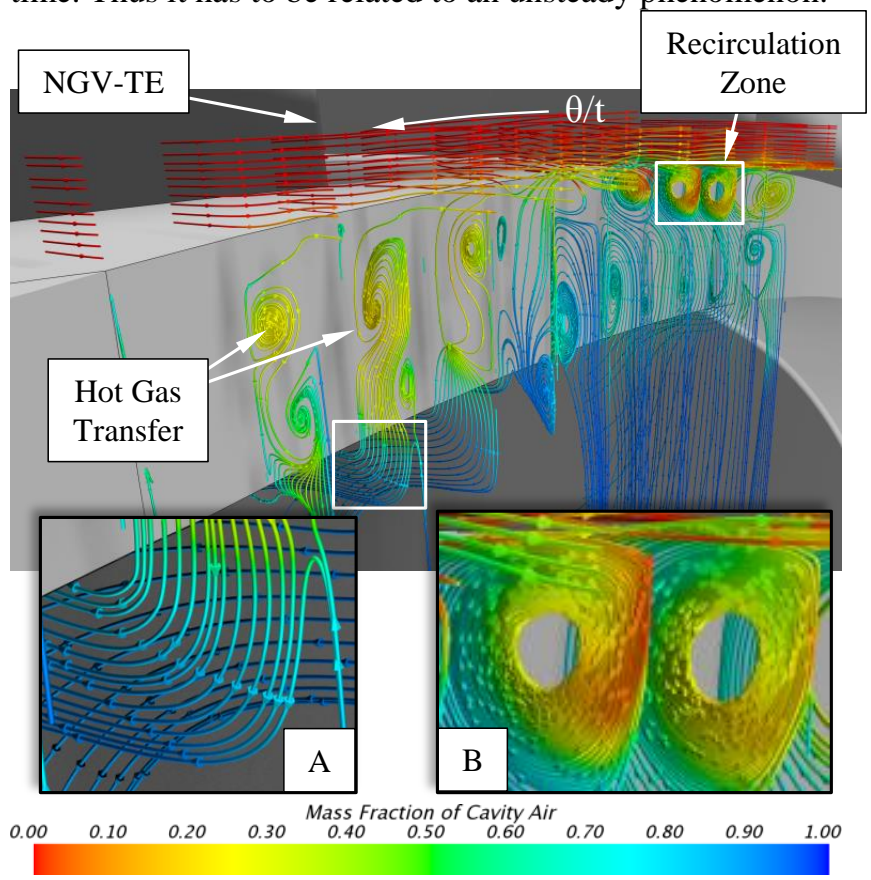

\section{Figure 10: Instant Seal flow field structure of the URANS calculation}

To further analyze the unsteady phenomenon in that area, a Fast Fourier Transform has been performed with the circumferential averaged pressure extracted at $r / R=0.981$ on the lip surface. The resulting amplitudes in dependency of the normalized frequency are plotted in Figure 11. The frequency is normalized with the blade passing frequency. Two frequencies happened to be dominant, of which one of them is the blade passing frequency itself. The other frequency is at 0.286 .

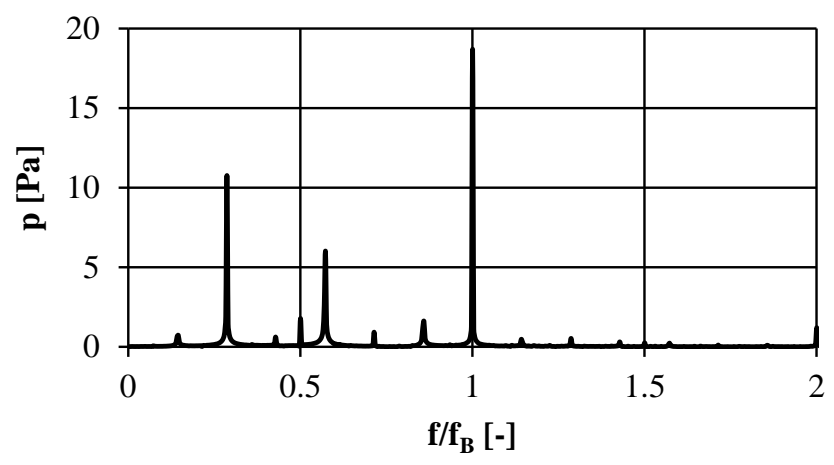

Figure 11: Pressure amplitudes against the normalized frequency at $r / R=0.981$

The pressure amplitudes of $\mathrm{f} / \mathrm{f}_{\mathrm{B}}=0.286$ have been extracted in between the rotor disk surface and the sealing lip, at exactly $50 \%$ of the clearance $s_{c}$ and visualized in Figure 12. One can see the absolute amplitude of the pressure 
fluctuations distributed on a disk. The sector surface extraction is patterned in circumferential direction and mirrors the original values. The hub radius $\mathrm{R}$ and lip radius $\mathrm{R}_{\mathrm{Lip}}$ is pointed out for orientation reasons in the detailed view. The full disk shows a radial pressure fluctuation inside the wheel space area as well as pressure fluctuations in the clearance area. The fluctuation in the clearance area shows a circumferential distribution, with its maximum near the hub radius.

The radial pressure mode inside the wheel space is most likely the natural acoustic frequency of its volume. By calculating the natural modes of the volume with a simplified Eq. (1), derived out of a common acoustic model to be found in textbooks like (Zucrow, et al., 1987) and (Rossing, 2007), the first radial acoustic mode is defined at $\mathrm{f}_{\mathrm{v}} / \mathrm{f}_{\mathrm{B}}=0.285$, which is close to the extracted frequency of the calculation.

$$
f_{V}=\frac{\alpha_{n m} a_{m}}{2 \pi R_{V}}
$$

Thus one can assume that the pressure fluctuation sourced in the sealing clearance triggers the acoustic mode inside the wheel space volume.

Pogorelov et al (Pogorelov, et al., 2018) performed at the Institute of Aerodynamics at RWTH Aachen University high fidelity LES calculation of this test case. They considered a full annulus model and observed two dominant frequencies of radial velocity fluctuations inside the seal gap. One of those frequencies was identified as the blade passing frequency. The second fluctuation, non-related to the blade harmonics, was identified as the driving ingestion source into the wheel space volume. The frequency was stated to be $f / n$ $\approx 18$, which is the ratio of the observed frequency $\mathrm{f}$ to the rotational frequency $\mathrm{n}$. This ratio is relatively close to the ratio $\mathrm{f} / \mathrm{n}=17.74$, which can be obtained from the URANS calculation presented in this paper.

The fluctuation captured in the URANS approach is constrained to a wave length of $2 \pi \mathrm{r} / 31$ since the domain only includes 1 Vane out of 31 . The resulting angular velocity of that fluctuation is $0.58 \omega$, which is similar to the angular fluid velocity $v_{\theta} \cdot 2 \pi \mathrm{r}$ in the seal gap. By observing the circumferential location of the pressure fluctuation in Figure 12, one can see that the fluctuation is located downstream of the NGV-TE. Taking those two observations into account, one can conclude that a convective phenomenon, which is not identified yet, causes a pressure fluctuation when interacting with the wake flow of the vane trailing edge. The interaction causes a further radial pressure fluctuation, spreading into the wheel space volume.

The behaviour between the convective fluctuation and the acoustic mode is not fully investigated yet. This calculation indicates that the convective fluctuation causes the acoustic response. But these phenomena could be a result of the equilibrium between the natural mode of the wheel space volume and the seal gap fluctuation. The seal gap fluctuation adjusts its wave length and angular fluid velocity to match the frequency of the natural acoustic mode. If that would be the case, increasing the wave length of the seal gap fluctuation, by increasing the calculation domain, could result into an increase in tangential velocity of the fluid inside the seal gap.

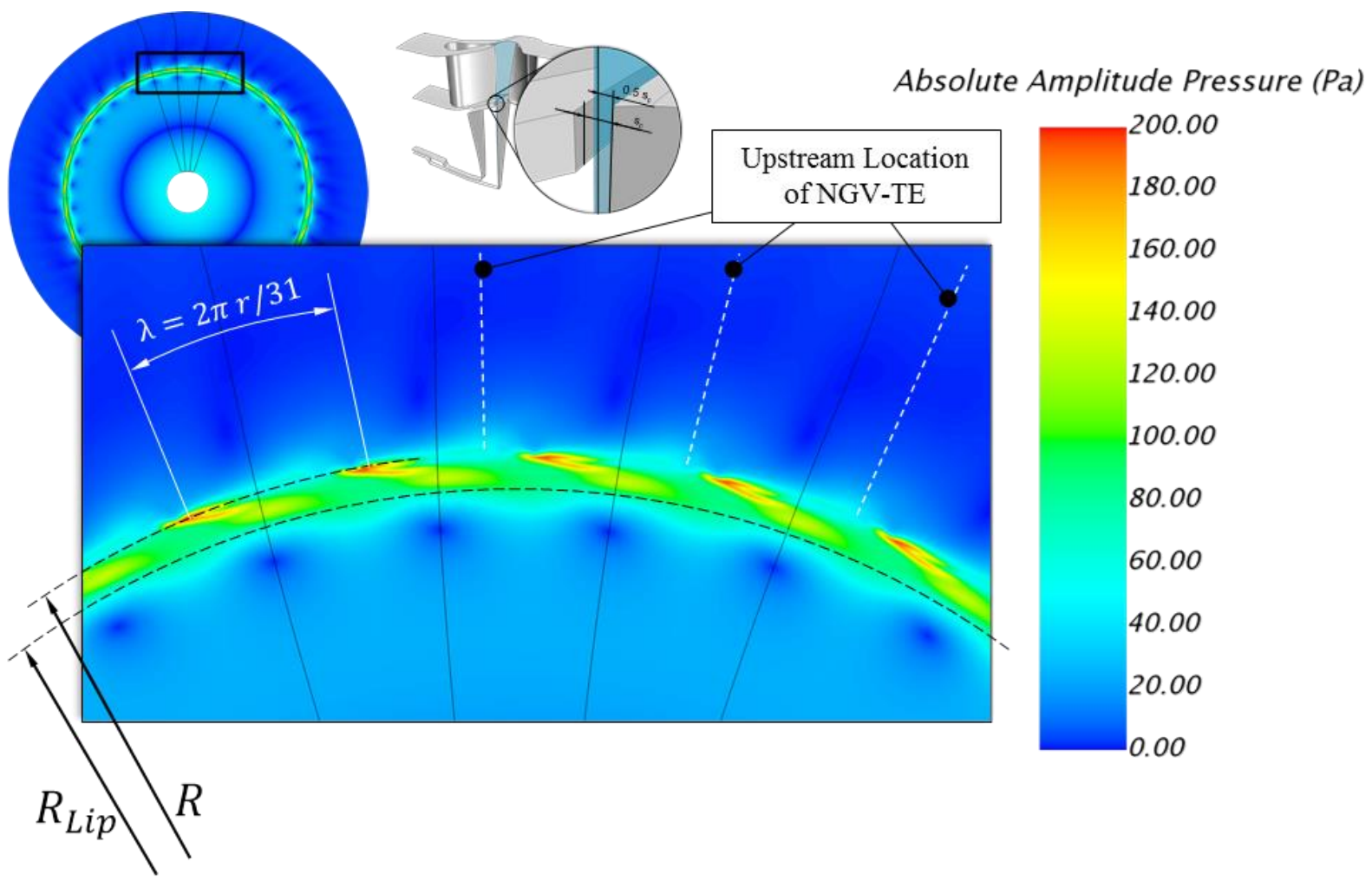

Figure 12: Pressure amplitudes of the normalized frequency 0.286 
Further could an increase of the calculation domain like a half or full model turbine, open up more potential variations of acoustic modes inside the wheel space volume. Tangential natural modes for example are more likely to capture in a full annulus domain, since the frequencies for a $2 \pi \mathrm{r} / 31$ domain and smaller are very high. An increased circumferential domain would free the fluctuation in the seal gap as well.

Since LES calculations by Pogorelov (Pogorelov, et al., 2018) were able to reproduce hot gas concentrations close to the measurements, the assumption is that the reduction to a sector model does limit the correct representation of the sealing performance for this test rig. But since the mesh uncertainty and the influence of the lower fidelity turbulence model cannot be excluded, further investigation with this sector model is needed. The unsteady RANS approach does predict more hot gas ingestion than the RANS simulation, but it is still weak in predicting the amount of ingestion and this concludes that the physics inside the seal gap does not represent the actual physics as well.

\section{CONCLUSION}

Considering the observation presented in this paper, it is safe to say that the demands in processing power for an unsteady RANS calculation for wheel space performance evaluations is still high. To run the simulation until convergence of the wheel space conditions, one needs to calculate many rotor rotations. Additionally the need of high mesh resolutions and assumingly larger sector models makes it even more expensive to perform.

To finalize the capability of the unsteady RANS calculation in predicting the hot gas ingress of this test configuration, additional investigation will be performed in the future. Calculations with meshes of higher resolutions and models with increased sector sizes and a full annulus domain need to be considered. Altogether, the URANS investigations are part of a comprehensive numerical approach to the topic including also the high fidelity LES work of the Institute of Aerodynamics at RWTH Aachen University. The aim is to derive funded conclusions in which regions of the flow field URANS simulations might have sufficient accuracy and where LES simulations are mandatory. Thus, the development of a zonal approach could provide improved results on such ingestion phenomena compared to a pure URANS simulation but still within an acceptable calculation effort compared to the high fidelity LES simulation.

\section{ACKNOWLEDGMENTS}

Simulations in this paper have been carried out with STAR-CCM+ of Siemens PLM. Their support is gratefully acknowledged.

\section{REFERENCES}

Bayley F. J. and Owen J. (1970). The Fluid Dynamics of a Shrouded Disk System With a Radial Outflow of Coolant. ASME Journal of Engineering and Power, pp. 335-341.

Bohn D. and Wolff M. (2003). Improved Formulation to Determine Minimum Sealing Flow - Cw, min - for Different Sealing Configurations. ASME Turbo Expo Atlanta, Georgia, USA.

Bohn D., Rudzinski B., Sürken N. and Gärtner W. (2000). Experimental and Numerical Investigation of the Influence of Rotor Blades on Hot Gas Ingestion into the Upstream Cavity of an Axial Turbine Stage. ASME Turbo Expo, Munich, Germany.

Boudet J., Hills N. J. and Chew J. W. (2006). Numerical Simulation of the Flow Interactions Between Turbin Main Annulus and Disc Cavities. ASME Turbo Expo, Barcelona, Spain.

Gentilhomme O., Hills N., Turner A. and Chew J. (2003). Measurement and analysis of ingestion through a turbine rim seal. ASME Journal of Turbomachinery, pp. 505-512.

Hamabe K. and Ishida K. (1992). Rim Seal Experiments and Analysis of a Rotor-Stator System With Nonaxisymmetric Main Flow. ASME 92-GT-160.

Jakoby R., Zierer T., Lindblad K., Larsson J., deVito L., Bohn D., Funcke J. and Decker A. (2004). Numerical Simulation of the Unsteady Flow Field in an Axial Gas Turbin Rim Seal Configuration. ASME Turbo Expo, Vienna, Austria.

Johnson B. V., Jakoby R., Bohn D. and Cunat D. (2009). A Method for Estimation the Influence of Time-Dependent Vane and Blade Pressure Fields on Turbine Rim Seal Ingestion. ASME Journal of Turbomachinery.

O'Mahoney T. S. D., Hills N. J., Chew J. W. and Timothy S. (2010). Large-Eddy Simulation of Rim Seal Ingestion. ASME Turbo Expo, Glasgow, UK.

Phadke U. P. and Owen J. M. (1988). Aerodynamic Aspects of the Sealing of Gas-Turbine Rotor-Stator Systems, Part 1: The Behaviour of Simple Shrouded Rotationg-Disk Systems in a Quiescent Environment. International Journal of Heat Fluid Flow, pp. 98-105, 1988.

Phadke U. P. and Owen J. M. (1988). Aerodynamic Aspects of the Sealing of Gas-Turbine Rotor-Stator Systems, Part 2: The Perfomance of Simple Seals in a Quasi Axisymmetric External Flow. International Journal of Heat Fluid Flow, pp. 106-112. 
Phadke U. P. and Owen J. M. (1988). Aerodynamic Aspects of the Sealing of Gas-Turbine Rotor-Stator Systems, Part 3: The Effect of Non-Axissymmetric External Flow on Seal Performance. International Journal of Heat Fluid Flow, pp. 113-117.

Porogelov A., Meinke M. and Wolfgang S. (2018). LargeEddy Simulation of Combined Ingress for Different Rim Seal Geometries. AIAA Aerospace Sciences Meeting, Kissimee, Florida.

Rabs M., Benra F.-K. and Schneider O. (2012). Emergence of Kelvin-Helmholtz Instabilities in Gas Turbine Rim Cavities - A Parameter Study. ASME Turbo Expo, Copenhagen, Denmark.

Rossing T., Springer Handbook of Acoustics (2007). New York, USA: Springer Science Business Media.

Zhou D. W., Wang C.-Z. and Glahn J. (2009). Main Gas Ingestion in a Turbine Stage for three Rim cavity Configurations. ASME Turbo Expo, Orlando, Florida, USA.

Zucrow M. J. and Hoffman J. D. (1987). Gas Dynamics, 2nd ed., vol. Volume 2, Krieger Publishing company. 